\title{
PMW: a Robust Clustering Algorithm for Mobile Ad-hoc Networks
}

\author{
Zhaowen Xing and Le Gruenwald \\ School of Computer Science \\ The University of Oklahoma, Norman, OK 73019, USA \\ \{zhaowenxing, ggruenwald\}@ou.edu
}

To mimic the operations in fixed infrastructures and to solve the routing scalability problem in large and dense Mobile Ad-hoc Networks (MANET), forming clusters of nodes where the operations in each cluster are managed by a node in the cluster, called cluster head, has been proven to be a promising approach. Many weighted clustering algorithms have been proposed to form clusters and select cluster heads. However, when calculating the weight utilized to determine whether a node is eligible to be a cluster head, these algorithms either consider only one metric (like mobility or power of nodes), or rely on some metric collected from extra devices (such as locations of nodes read from Global Positioning Systems). This leads to a high possibility of re-clustering and, consequently, quality of service cannot be provided.

This paper presents a novel weighted clustering algorithm, called PMW, to form and maintain more stable clusters in MANETs. In PMW, in order to take into account the major factors that frequently cause the network topology to change and in order to avoid being dependent on any extra devices, the weight of a node is calculated by three parameters: Power, Mobility and Workload (PMW), where:

- $\quad$ Power is the remaining battery power when the cluster head election takes place. A node with higher remaining power (RP) among neighbors is of course a better candidate for the cluster head. But, a higher RP shows only the current power level and whether it is still high in the future is a question, thus, the workload of each node has to be considered.

- $\quad$ Mobility is based on two metrics: relative mobility (RM) [Basu, 2001] and mobility prediction (MP). RM is the ratio of the received signal strength read from two successive receptions of hello packets sent by the same neighbors, where the received signal strength is getting smaller when the neighbors move away. MP is the standard deviation of all the neighbors' RMs, such that the mobility is captured in everyone's neighborhood.

- $\quad$ Workload is measured by the power decrease rate (PDR) during a time interval. A node with less PDR over a large time interval in the history will most likely have less workload in the future.

Cluster formation: after collecting the values of RP, MP and PDR, the weight of each node $j$ is calculated by a normalized formula and broadcast: $W_{j}=\frac{f_{1}}{e^{M P_{j}}}+f_{2} * R P_{j}+\frac{f_{3}}{e^{P D R_{j}}}$, where $\mathrm{f}_{1}, \mathrm{f}_{2}$ and $\mathrm{f}_{3}$ are weighting factors and can be set according to applications, and their sum is 1 . After receiving the weights from all neighbors, the nodes with the largest weight are elected as the cluster heads, and their 1-hop neighbors just join them as members. No two cluster heads should be 1-hop neighbors.

Cluster maintenance: When a member/cluster head moves out of the current cluster, it will either join another cluster or declare itself as a cluster head. To reduce the computation and communication cost, once a cluster head resigns due to node mobility or power drainage, the reelection for the replacement is only triggered inside the current cluster.

To evaluate the performance of PMW, the simulation is performed using NS-2 simulator. We compare PMW with MOBIC [Basu, 2001] and SCA [Sheu, 2006] in terms of lifetime of clusters and average number of cluster head changes.

\section{References:}

[Basu, 2001] P. Basu, N. Khan and T. D. C. Little. "A Mobility Based Metric for Clustering in Mobile Ad Hoc Networks" In Proceeding of IEEE ICDC, April, 2001, pp. 413-418.

[Sheu, 2006] P. Sheu and C. Wang, "A Stable Clustering Algorithm Based on Battery Power for Mobile Ad Hoc Networks", Tamkang Journal of Science and Engineering, Vol. 9, Num. 3, 2006, pp. 233-242. 